\title{
Current innovation in virtual and augmented reality in spine surgery
}

\author{
Frank J. Yuk ${ }^{1}$, Georgios A. Maragkos ${ }^{1}$, Kosuke Sato $^{2}$, Jeremy Steinberger ${ }^{1}$ \\ ${ }^{1}$ Department of Neurosurgery, Mount Sinai Hospital, Icahn School of Medicine at Mount Sinai, New York, NY, USA; ${ }^{2}$ Hospital for Special Surgery, \\ New York, NY, USA \\ Contributions: (I) Conception and design: FJ Yuk, J Steinberger; (II) Administrative support: All authors; (III) Provision of study materials or patients: \\ FJ Yuk, J Steinberger; (IV) Collection and assembly of data: FJ Yuk, GA Maragkos; (V) Data analysis and interpretation: FJ Yuk, GA Maragkos, K \\ Sato; (VI) Manuscript writing: All authors; (VII) Final approval of manuscript: All authors. \\ Correspondence to: Frank J. Yuk, MD. Department of Neurosurgery, 1468 Madison Ave, Annenberg building 8-03, New York, NY 10029, USA. \\ Email: Frank.yuk@mountsinai.org.
}

\begin{abstract}
In spinal surgery, outcomes are directly related both to patient and procedure selection, as well as the accuracy and precision of instrumentation placed. Poorly placed instrumentation can lead to spinal cord, nerve root or vascular injury. Traditionally, spine surgery was performed by open methods and placement of instrumentation under direct visualization. However, minimally invasive surgery (MIS) has seen substantial advances in spine, with an ever-increasing range of indications and procedures. For these reasons, novel methods to visualize anatomy and precisely guide surgery, such as intraoperative navigation, are extremely useful in this field. In this review, we present the recent advances and innovations utilizing simulation methods in spine surgery. The application of these techniques is still relatively new, however quickly being integrated in and outside the operating room. These include virtual reality (VR) (where the entire simulation is virtual), mixed reality (MR) (a combination of virtual and physical components), and augmented reality (AR) (the superimposition of a virtual component onto physical reality). VR and MR have primarily found applications in a teaching and preparatory role, while AR is mainly applied in hands-on surgical settings. The present review attempts to provide an overview of the latest advances and applications of these methods in the neurosurgical spine setting.
\end{abstract}

Keywords: Spine surgery; neurosurgery; orthopaedic surgery; virtual reality (VR); augmented reality (AR); mixed reality $(\mathrm{MR})$

Submitted Jan 30, 2020. Accepted for publication Jun 11, 2020.

doi: $10.21037 / \mathrm{atm}-20-1132$

View this article at: http://dx.doi.org/10.21037/atm-20-1132

\section{Introduction}

New advances in spine surgery such as augmented reality (AR) and virtual reality (VR) are becoming more common as both operative and teaching tools. The applications and uses are still relatively new and are in constant evolution. Currently, there are three types of simulation systems employed. The first is VR, where the entire simulation is virtual. The second is AR, a technology that superimposed a computer generated image onto the view of the real world and lastly is mixed reality (MR), which combines both virtual and physical experiences. AR in spine surgery has been studied in cases throughout the entire spine, however
VR simulator have mostly been reserved for teaching of residents and medical students. We describe the recent trends in the use of VR, MR, and AR simulators in spine surgery. The goal of these modalities is to offer more precise surgical results and improve clinical outcomes.

\section{Methods}

A PUBMED and MEDLINE database search was conducted to explore the current literature that focused on $\mathrm{VR}$ and $\mathrm{AR}$ and its intraoperative use in the operating room for spine surgery. In the database searches "spine surgery" was combined with "virtual reality", "augmented reality", 
"mixed reality", "virtual fluoroscopy", "intraoperative image guidance", "simulation", and "intraoperative navigation". We included randomized clinical trials, comparative studies, case studies published from 2010 to 2020 .

\section{Discussion}

The accuracy of transpedicular screw placement has long been a central concern in spine surgery. Violation of the cortical bone of the pedicles or vertebral bodies can lead to spinal, nerve root, or vascular injury, as well as dural tears and cerebrospinal fluid (CSF) leaks. Additionally, screw misplacement can lead to inadequate purchase and fusion failure. These risks are even more critical in the narrow pedicles of the cervical and thoracic spine, as well as in cases of complex rotational spinal deformity or prior surgery.

\section{Fluoroscopy-assisted navigation}

Fluoroscopy-assisted navigation has received much attention as a straightforward way to improve screw placement accuracy, however has a high barrier of entry as intraoperative CT scanners and associated navigation systems are costly. Innocenzi et al. assessed the potential benefits of using navigation in thoracolumbar pedicular screw placement (1). Specifically, they compared the efficacy of an open free-hand technique, an open navigated technique (BrainLab ${ }^{\circledR}$ System), a percutaneous CT-based navigation technique, and a percutaneous fluoroscopyguided technique. They concluded that open, navigationassisted pedicular screw placement was significantly more accurate and less likely to cause a breech than the other techniques. In an earlier series, Luther et al. placed 708 screws in the cervical, thoracic or lumbar spine using $3 \mathrm{D}$ NAV guidance and 726 using the free-hand technique and found the former to have significantly better screw placement accuracy, and allow for both higher screw-topedicle diameter ratios, as well as more complex cases (2).

Aoude $e t$ al. performed a meta-analysis of articles published between 2010 and 2013 regarding screw fixation classification schemes (3). The most commonly utilized classification was based on 2-mm breech increments. The second most commonly used classification is the "in" or "out" breech assessment. The authors concluded that, using both classification schemes, navigation-assisted pedicle screw placement had statistically significantly less breech cases than free-hand technique. In an earlier metaanalysis, Mason et al. assessed the screw placement accuracy differences between navigation systems (4). They concluded that $3 \mathrm{D}$ fluoroscopic navigation was significantly more accurate for pedicle screw placement than conventional fluoroscopy or 2D fluoroscopic navigation to demonstrate an aggregate screw placement accuracy of $68.1 \%$ with conventional fluoroscopy, $84.3 \%$ with 2D fluoroscopic guidance and $95.5 \%$ with $3 \mathrm{D}$ fluoroscopic navigation.

\section{$V R$}

VR is gaining traction in the education and training of anatomy, as demonstrated by the $3 \mathrm{D}$ neuroanatomy atlas by Aaron-Cohen Gado (5). Furthermore, many authors are exploring its application in an educational and preparational function, providing medical students and residents with an opportunity to practice surgical procedures in a controlled environment before participating in actual surgery. The main benefit of this training paradigm is the opportunity to improve one's surgical technique, while minimizing the possibility of making a mistake on a live patient, which could result in devastating outcomes, therefore these systems are often cost effective.

\section{Resident training}

In a study of novice orthopedic residents who were practicing cervical pedicle screw placements on cadavers, the potential advantages of VR compared to traditional methods of learning were explored (6). This study demonstrated that the VR-trained group outperformed the control in all areas, including screw penetration rates (10\% vs. $62.5 \%$, respectively), acceptable screw placement rates $(100 \%$ vs. $50 \%)$, as well as mean screw penetration distance (1.12 vs. $2.08 \mathrm{~mm}$ ). The authors concluded that VR training tool can improve performance of novice residents in cervical pedicle screw placement. The same authors performed a similar experiment comparing junior residents with and without VR training in the placement of pedicular screws in the thoracic spine in cadavers (T6-T12), again finding significantly better and safer placement in the VR-trained group in all parameters (7). A separate group explored VR training for thoracolumbar screw placement (T11-L4 pedicle screws), again demonstrating the benefits of this training paradigm (8). As such, the usefulness of VR training for resident training for pedicular screw placement has been demonstrated by multiple groups.

\section{Adjunct for experienced spine surgeons}

In an effort to expand the uses of VR into the practice 
of experienced spine surgeons, Zhou et al. explored its utility combined with isocentric navigation for minimally invasive lumbar transforaminal microdiscectomy (9). In this study, the same surgeon placed L3-S1 screws on the left side of a cadaver without the use of $\mathrm{VR}$ and isocentric navigation, then had a session of VR simulation for surgical planning, then placed screws on the other side. The VR-planned side had better exposure and punctureto-channel times. In a similar vein, Zheng et al. compared percutaneous endoscopic lumbar discectomies with and without preoperative VR planning for thirty randomized patients, and found improved fluoroscopic and location timing when VR planning was used (10). VR may have a place for preoperative planning in minimally invasive spinal procedures, especially in more complex cases.

\section{D assessment of imaging}

VR has been suggested as a novel modality to view complex spine conditions. Lafage et al. explored the use of VR for comparing preoperative and postoperative imaging of patients with adult spinal deformity (11). Their main focus was to account for proximal junctional kyphosis (which can cause multiple alignment changes), in order to eliminate its confounding effects on the evaluation of sagittal alignment after surgical correction. They demonstrate that the use of VR for 3D reconstruction can greatly benefit this assessment and potentially assist in management decision-making. Zawy Alsofy et al. evaluated the potential influence of VR on surgical planning for patients with cervical neuroforaminal stenosis (12). While they concluded that VR technique can be helpful in planning surgical approaches and treatment strategies, the influence of VR was judged as moderate. With more dramatic pathology (massive herniated disc, complex spinal deformity, traumatic 3 column injury, etc.), VR modeling can demonstrate pathology with ease, however, with more subtle findings (compression of the neural foramen, low grade spondylolisthesis), VR has yet to demonstrate excellent utility.

\section{Applications in rehabilitation}

Apart from its uses in training and preoperative planning, it should be noted that VR has seen utility in rehabilitation of patients with spine pathology with promising early results. Sengupta et al. demonstrated that VR gaming can be a useful rehabilitation adjunct for spinal cord injury patients (13). Maresca et al. expanded on the concept with a case report of a complicated patient who seemed to benefit from VR in balance, motor and cognitive skills, when combined with traditional rehabilitation protocols (14). These are novel and exciting opportunities to improve patient function and assist in patient outcomes, that call for more research and efforts to further explore their utility in the future.

\section{$M R$}

One of the most important benefits of simulation training is that it provides trainees with "permission to fail" in a safe environment without compromising patient safety. As discussed above, however, VR is an entirely virtual experience, providing trainees with visual but no mechanical feedback. MR, on the other hand, is the combination of a virtual environment with haptic feedback, usually in the form of a physical model. In this regard, surgical training can be better facilitated with incorporation of muscle memory training. Spine surgery, in particular, is a field that relies heavily on haptic feedback for the correct placement of pedicular screws and percutaneous procedures, and could benefit from MR training.

Coelho et al. evaluated a lumbar spine fusion simulation model combining a virtual and a physical component (15). The physical model is made of synthetic materials designed to produce textures, consistencies, and mechanical resistance similar to human tissues, allowing for the practice of pedicle screw placement and lumbar stenosis decompression. Specifically, the model facilitates simulation of the skin incision, subcutaneous, muscular and subperiosteal dissection, laminectomy, and placement of the pedicle screws as well as the management of intraoperative complications such as CSF leaks. Unfortunately, the virtual simulator was not designed to be superimposed on the physical model, rather it is to be used as an adjunct. Sixteen spine surgeons in Brazil evaluated the model and responded to a questionnaire regarding the model's accuracy and perceived utility in training neurosurgery residents, coming to a conclusion that $94 \%$ of the participants felt that the simulator was an appropriate field for training residents. In a similar vein, Stefan et al. created a MR model combining a $3 \mathrm{D}$ printed vertebral phantom inside an agar gel with an overhead monitor displaying $\mathrm{X}$-rays of the model depending on the orientation of a C-arm (16). In this way, the trainee can practice percutaneous pedicular screw placement in a fairly realistic operating room setting, without being subjected to radiation. Multiple surgeons agreed that this concept can be useful for the training of both residents and surgeons alike.

Notably, the study design of these studies implies a 
degree of subjectivity in the assessment of the MR models, bringing their validity and generalizability into question. Nevertheless, the concept of combining virtual and physical models for surgical simulation can help address the shortcomings of the traditional paradigm of apprenticeshipbased training by giving residents the chance to execute critical procedures and gain muscle memory skills, before attempting them on real patients. Furthermore, the use of synthetic training models in lieu of cadaveric dissection can help overcome multiple shortcomings of the latter, including inconsistencies in anatomy of dead versus living tissue-which can be an obstacle in replicating fine operative manipulations-as well as the lack of specific pathologies, ethical and economic implications related to cadaveric preservation and dissection.

\section{AR}

AR can capture real objects in real time with virtual objects are overlaid onto the image. This is similar to a pilot's ability to have heads up display, with relevant flight safety information projected onto their view from the cockpit. In spine surgery, AR has been utilized to minimize fluoroscopy and radiation exposure to both the patient and the treatment team, as well as to reduce procedural time, however these systems are fairly new and costly making it difficult to access.

\section{Pedicle screw placement}

While fluoroscopic navigation has been shown to improve placement accuracy compared to the freehand technique, it remains time-consuming and subjects both the patient and the treatment team to radiation exposure. Recent advances in AR have enabled the production of precise optical seethrough head-mounted displays (HMD), which could potentially be used for the navigation of pedicle screws. Edström et al. developed a standardized workflow for ARassisted surgical navigation for pedicle screw placement (17). Their suggested plan includes intraoperative cone-beam $\mathrm{CT}$ for AR registration and was tested in 20 patients, with a mean total time of 279 min per procedure.

Overall, it seems that AR-assisted pedicle screw placement can offer better accuracy than the free-hand technique. Elmi-Terander et al. compared the use of AR-assisted pedicle screw placement in the thoracic spine against the free-hand technique without the use of fluoroscopy in cadavers (18). They found improved overall accuracy, higher rates of perfectly placed screws, and lower rates of 4-cm breech with AR. After their cadaveric study, the same authors applied their AR-assisted technique to place thoracic and lumbosacral pedicular screws in 20 patients, demonstrating acceptable accuracy, breech rates and timing, without the use of fluoroscopic guidance (19). When compared to the fluoroscopy-assisted paradigm, accuracy is similar, with AR having less radiation exposure and decreased timing. Urakov et al. compared fluoroscopyassisted screw placement with AR-assisted placement and emphasized the importance of an accurate registration process (20).

While these techniques have been developed to avoid using intraoperative fluoroscopy, the patient still has to be exposed to an intraoperative cone-beam CT to register the AR. To avoid that, Ma et al. proposed a technique of AR-assisted pedicle screw placement utilizing ultrasound instead of CT guidance for registration (21). They demonstrated that their proposed method had higher accuracy in AR-assisted screw placement than skin markersbased registration. Liebman et al. expanded on that concept by developing a navigation method to run on an HMD (Microsoft HoloLens, Microsoft corp., Redmond, WA, USA), based on intraoperative capturing of the reachable surfaces of the vertebrae for registration (22). Then they used a vertebral phantom to test their algorithm, with promising results. The main advantage of this technique is that it requires no intraoperative imaging, either in the form of fluoroscopy or intraoperative CT. Initial registration is based on the preoperative CT scan and intraoperatively the system is registered on the actual vertebrae after surgical exposure. This concept could help to further improve operative times and reduce radiation exposure.

\section{Rod bending}

Wanivenhaus et al. developed an AR application for accurate rod bending in spine surgery (23). Using the Microsoft HoloLens HMD, the operator looks at the already placed pedicle screw heads, which are then registered in $3 \mathrm{D}$ space using fiducials. Subsequently, a hologram of the ideal rod is presented to the operator's view, which can be used as a guiding point for rod bending.

\section{Minimally invasive pedicle screws}

Expanding from navigation in open spine surgery, Burström et al. studied the accuracy of minimally invasive pedicle screw placement using AR instead of fluoroscopy (24). The technique was tested in two pig cadavers, demonstrating acceptable technical accuracy both in entry 
point ascertainment and in angulation and required no further radiation exposure other than the initial CT. Gibby et al. utilized an opaque lumbar spine model to assess the utility of an HMD AR model (Microsoft HoloLens) with superimposed computed tomography (CT) data in percutaneously placing pedicle screws, again with no realtime fluoroscopic guidance (25). It took an average of 200 seconds to place the percutaneous needles and 97\% of them were accurately placed. In a similar way, ElmiTerander $e t$ al. also utilized AR to assess screw placement accuracy and navigation time for minimally invasive spine surgery (26). They reported that navigation time per insertion was around 90 seconds, with acceptable accuracy. They concluded that minimally invasive spine screw placement using AR is accurate and efficient without radiation exposure during the procedure.

\section{Intraoperative quality assurance}

Instead of an AR-based navigation system for pedicle screw placement, Uneri et al. turned towards intraoperative quality assurance by ensuring correct screw placement during surgery. They developed an intraoperative 3D-2D registration method, combined with $3 \mathrm{D}$ models of known surgical devices, such as pedicle screws, aiming to provide a near-real-time assessment of pedicle breech for quality assurance purposes (27). This can help surgeons identify breech or mispositioning more easily, since 3D images may be better perceived and assessed than anteroposterior and lateral X-rays taken after placement.

\section{Percutaneous vertebroplasty}

Another minimally invasive application for AR guidance is percutaneous vertebroplasties. In these procedures, the main concern is accurate placement of the needle through a planned trajectory into the vertebral body. Abe et al. developed a virtual protractor with augmented reality (VIPAR) system to visualize the needle trajectory in threedimensional space during percutaneous vertebroplasty (28). The system makes use of a heads-up display (HUD), which overlays the preoperatively planned needle trajectory onto the operative field based on a marker on the patient detected using a tracking camera. The model was validated in 40 spine phantom trials showing significantly less error in needle insertion angle when using the system compared to not using it and was then utilized in 5 patients with osteoporotic vertebral fractures with low angle errors and no breech or leakage. Auloge et al. developed a tool integrating artificial intelligence and AR for needle placement in percutaneous vertebroplasty for compression fractures (29). They performed a prospective, randomized, open label trial comparing this technique to the fluoroscopy-assisted standard and found similar accuracy and clinical outcomes but significant less radiation exposure in the AI/AR group. Percutaneous vertebroplasty seems to be a great candidate for the application of AR guidance, with the main endpoint of less radiation exposure.

\section{Keyhole spine surgery}

A model has also been described to utilize AR for keyhole spine surgery (30). The authors used $\mathrm{O}$-arm AR registration for a case of transvertebral anterior cervical foraminotomy and a case of posterior foraminotomy. In both cases they displayed the AR imaging model on the surgical microscope view. HUD in this manner has been previously utilized in brain surgery and has many proponents. In keyhole spine surgery, full anatomic exposure to identify relevant landmarks is inherently not attempted, therefore it may significantly benefit from this strategy, without the limitations of brain shift leading to registration inaccuracies.

\section{Displaying imaging on side vision}

One distinct disadvantage of overlaying holograms on the actual anatomy is that many users find this to be too cluttered, opting to disable the feature at various points during the procedure. Deib et al. also explored the use of see-through HMDs in MIS spine, but instead of overlaying registered images onto the anatomy, they opted to display anteroposterior and lateral radiographs on the side view of the operator (31). In this way, they attempted to reduce the amount of movement needed to see imaging on monitors, eliminating the need to take one's eyes off the operative field. They propose that with this method there is less clutter during visualization of the patient's anatomy, while retaining a large amount of information readily available for the proceduralist. Similarly, Yoon et al. used the Google Glass HUD to assist in placing pedicle screws in 10 live surgeries (32). In this way, they demonstrated a $15 \%$ decrease in operating time, from 4.86 to 4.13 minutes.

\section{Facet joint injections}

Agten et al. utilized an AR HMD (Microsoft HoloLens) to display a $3 \mathrm{D}$ spine phantom on a physical model and assessed the accuracy of lumbar facet joint injections done by radiologists under such display (33). They found significantly improved timing and acceptable needle placement compared to the standard CT-guided needle 
placement. The prospect of decreased radiation exposure as well as procedural time is very appealing for such procedures that may need to be repeated multiple times.

\section{Workflows for registration}

Carl et al. developed and tested a workflow for AR registration and integration in spine surgery (34). This is based on intraoperative CT scanning for automated registration and subsequent HUD of the vertebrae, tumor extent, and instrumentation on the surgical microscope. The same authors showed that the techniques currently employed in cranial surgery for HUD can be applied to spine tumor surgery (35). In a separate publication, they demonstrated that intraoperative CT can be used with a low-dose protocol for AR registration in spine cases with registration errors of less than $1 \mathrm{~cm}$ and $70 \%$ decrease in overall radiation exposure, which can further facilitate AR usage in spine surgery (36). Müller et al. explored the use of 3D intraoperative fluoroscopy for AR registration (37). They then used cadavers to compare drilling accuracy between the AR-assisted technique and the gold standard with pose-tracking system. They found no significant difference between the two methods, demonstrating that holographic navigation achieves accuracy similar to the gold standard. The use of lower radiation protocols for AR registration is an important step towards decreased radiation exposure, which is a central goal of AR-guided surgery.

\section{Previous reviews}

Novel simulation systems such as VR, MR and AR have primarily been used for brain surgery over the past few years, while spine surgery is now seeing progressively more use for such imaging modalities. In a 2018 review by Yoon et al. regarding the use of AR in any surgical setting (38), a total of five neurosurgical studies were identified using a HUD in live surgery and another five using it for simulation. The technology was found to be used primarily in cranial surgery and for educational purposes. They maintain that the main benefits of HUD $\mathrm{AR}$ are ergonomics, multitasking and the ability to record in the first person, while it is mainly limited by small projection dimensions, battery life and possibly patient privacy. Similarly, in their systematic review between 2000 and 2015, Guha et al. investigated the utility of AR in the neurosurgical setting. They found that most of the literature at that time had focused on its applications in skull base, vascular and functional neurosurgery, with limited studies on AR-assisted spine surgery (39).

In their recent review of the literature, Chakravarthy et al. discuss the historical evolution of imaging technologies in spine surgery and comment on the more recent integration of $A R, V R$ and $M R$, especially in pedicle screw navigation (40). Moreover, in a 2017 systematic literature review on VR, MR and AR in spine surgery, Pfandler et al. concluded that, while these systems have been promoted and researched for many procedures, including pedicle screw placement, vertebroplasty, posterior cervical laminectomy and foraminotomy, lumbar puncture, facet joint injection, and spinal needle insertion (41), there is paucity for data related to actual patient care and patient outcomes. Randomized controlled trials evaluating clinical performance and patient outcomes are required to demonstrate the effectiveness of VR-based simulators in spinal surgery.

\section{Conclusions}

This review demonstrates the current state of VR, MR, and AR simulators currently used in spine surgery today. Educating new trainees with VR appears to be an effective method of teaching both traditional and new methods of spine surgery, however it cannot replace training on real patients. It should be used as a supplement rather than a training replacement for real patients. AR simulators again seem to be an effective adjunct to the current efforts in spine surgery, however randomized controlled trials are still required to evaluate accuracy, cost-effectiveness, and patient outcomes. With curiosity, collaboration, and innovation, the uses of $\mathrm{VR}$ and $\mathrm{AR}$ will undoubtedly increase.

\section{Acknowledgments}

Funding: None.

\section{Footnote}

Provenance and Peer Review: This article was commissioned by the Guest Editor (Dr. Sheeraz Qureshi) for the series "Current State of Intraoperative Imaging" published in Annals of Translational Medicine. The article was sent for external peer review organized by the Guest Editor and the editorial office.

Conflicts of Interest: All authors have completed the ICMJE uniform disclosure form (available at http://dx.doi. 
org/10.21037/atm-20-1132). The series "Current State of Intraoperative Imaging" was commissioned by the editorial office without any funding or sponsorship. The authors have no other conflicts of interest to declare.

Ethical Statement: The authors are accountable for all aspects of the work in ensuring that questions related to the accuracy or integrity of any part of the work are appropriately investigated and resolved.

Open Access Statement: This is an Open Access article distributed in accordance with the Creative Commons Attribution-NonCommercial-NoDerivs 4.0 International License (CC BY-NC-ND 4.0), which permits the noncommercial replication and distribution of the article with the strict proviso that no changes or edits are made and the original work is properly cited (including links to both the formal publication through the relevant DOI and the license). See: https://creativecommons.org/licenses/by-nc-nd/4.0/.

\section{References}

1. Innocenzi G, Bistazzoni S, D'Ercole M, et al. Does Navigation Improve Pedicle Screw Placement Accuracy? Comparison Between Navigated and Non-navigated Percutaneous and Open Fixations. Acta Neurochir Suppl 2017;124:289-95.

2. Luther N, Iorgulescu JB, Geannette C, et al. Comparison of navigated versus non-navigated pedicle screw placement in 260 patients and 1434 screws: screw accuracy, screw size, and the complexity of surgery. J Spinal Disord Tech 2015;28:E298-303.

3. Aoude AA, Fortin M, Figueiredo R, et al. Methods to determine pedicle screw placement accuracy in spine surgery: a systematic review. Eur Spine J 2015;24:990-1004.

4. Mason A, Paulsen R, Babuska JM, et al. The accuracy of pedicle screw placement using intraoperative image guidance systems. J Neurosurg Spine 2014;20:196-203.

5. Tomlinson SB, Hendricks BK, Cohen-Gadol A. Immersive Three-Dimensional Modeling and Virtual Reality for Enhanced Visualization of Operative Neurosurgical Anatomy. World Neurosurg 2019;131:313-20.

6. Hou Y, Shi J, Lin Y, et al. Virtual surgery simulation versus traditional approaches in training of residents in cervical pedicle screw placement. Arch Orthop Trauma Surg 2018;138:777-82.

7. Hou Y, Lin Y, Shi J, et al. Effectiveness of the Thoracic Pedicle Screw Placement Using the Virtual Surgical
Training System: A Cadaver Study. Oper Neurosurg (Hagerstown) 2018;15:677-85.

8. Xin B, Chen G, Wang Y, et al. The Efficacy of Immersive Virtual Reality Surgical Simulator Training for Pedicle Screw Placement: A Randomized Double-Blind Controlled Trial. World Neurosurg 2018;S1878-8750(18)32913-9.

9. Zhou Z, Hu S, Zhao YZ, et al. Feasibility of Virtual Reality Combined with Isocentric Navigation in Transforaminal Percutaneous Endoscopic Discectomy: A Cadaver Study. Orthop Surg 2019;11:493-9.

10. Zheng C, Li J, Zeng G, et al. Development of a Virtual Reality Preoperative Planning System for Postlateral Endoscopic Lumbar Discectomy Surgery and Its Clinical Application. World Neurosurg 2019;123:e1-8.

11. Lafage R, Bess S, Glassman S, et al. Virtual Modeling of Postoperative Alignment After Adult Spinal Deformity Surgery Helps Predict Associations Between Compensatory Spinopelvic Alignment Changes, Overcorrection, and Proximal Junctional Kyphosis. Spine (Phila Pa 1976) 2017;42:E1119-25.

12. Zawy Alsofy S, Stroop R, Fusek I, et al. Virtual RealityBased Evaluation of Surgical Planning and Outcome of Monosegmental, Unilateral Cervical Foraminal Stenosis. World Neurosurg 2019;129:e857-65.

13. Sengupta M, Gupta A, Khanna M, et al. Role of Virtual Reality in Balance Training in Patients with Spinal Cord Injury: A Prospective Comparative Pre-Post Study. Asian Spine J 2020;14:51-8.

14. Maresca G, Maggio MG, Buda A, et al. A novel use of virtual reality in the treatment of cognitive and motor deficit in spinal cord injury: A case report. Medicine (Baltimore) 2018;97:e13559.

15. Coelho G, Defino HLA. The Role of Mixed Reality Simulation for Surgical Training in Spine: Phase 1 Validation. Spine (Phila Pa 1976) 2018;43:1609-16.

16. Stefan P, Pfandler M, Wucherer P, et al. Team training and assessment in mixed reality-based simulated operating room: Current state of research in the field of simulation in spine surgery exemplified by the ATMEOS project. Unfallchirurg 2018;121:271-7.

17. Edström E, Burstrom G, Nachabe R, et al. A Novel Augmented-Reality-Based Surgical Navigation System for Spine Surgery in a Hybrid Operating Room: Design, Workflow, and Clinical Applications. Oper Neurosurg (Hagerstown) 2020;18:496-502.

18. Elmi-Terander A, Skulason H, Soderman M, et al. Surgical Navigation Technology Based on Augmented Reality and Integrated 3D Intraoperative Imaging: A Spine 
Cadaveric Feasibility and Accuracy Study. Spine (Phila Pa 1976) 2016;41:E1303-11.

19. Elmi-Terander A, Burstrom G, Nachabe R, et al. Pedicle Screw Placement Using Augmented Reality Surgical Navigation With Intraoperative 3D Imaging: A First InHuman Prospective Cohort Study. Spine (Phila Pa 1976) 2019;44:517-25.

20. Urakov TM, Wang MY, Levi AD. Workflow Caveats in Augmented Reality-Assisted Pedicle Instrumentation: Cadaver Lab. World Neurosurg 2019;126:e1449-55.

21. Ma L, Zhao Z, Chen F, et al. Augmented reality surgical navigation with ultrasound-assisted registration for pedicle screw placement: a pilot study. Int J Comput Assist Radiol Surg 2017;12:2205-15.

22. Liebmann F, Roner S, von Atzigen M, et al. Pedicle screw navigation using surface digitization on the Microsoft HoloLens. Int J Comput Assist Radiol Surg 2019;14:1157-65.

23. Wanivenhaus F, Neuhaus C, Liebmann F, et al. Augmented reality-assisted rod bending in spinal surgery. Spine J 2019;19:1687-9.

24. Burström G, Nachabe R, Persson O, et al. Augmented and Virtual Reality Instrument Tracking for Minimally Invasive Spine Surgery: A Feasibility and Accuracy Study. Spine (Phila Pa 1976) 2019;44:1097-104.

25. Gibby JT, Swenson SA, Cvetko S, et al. Head-mounted display augmented reality to guide pedicle screw placement utilizing computed tomography. Int J Comput Assist Radiol Surg 2019;14:525-35.

26. Elmi-Terander A, Nachabe R, Skulason H, et al. Feasibility and Accuracy of Thoracolumbar Minimally Invasive Pedicle Screw Placement With Augmented Reality Navigation Technology. Spine (Phila Pa 1976) 2018;43:1018-23.

27. Uneri A, De Silva T, Stayman JW, et al. Knowncomponent 3D-2D registration for quality assurance of spine surgery pedicle screw placement. Phys Med Biol 2015;60:8007-24.

28. Abe $\mathrm{Y}$, Sato S, Kato K, et al. A novel 3D guidance system using augmented reality for percutaneous vertebroplasty: technical note. J Neurosurg Spine 2013;19:492-501.

29. Auloge P, Cazzato RL, Ramamurthy N, et al. Augmented reality and artificial intelligence-based navigation during percutaneous vertebroplasty: a pilot randomised clinical trial. Eur Spine J 2020;29:1580-9.

30. Umebayashi D, Yamamoto Y, Nakajima Y, et al.
Augmented Reality Visualization-guided Microscopic Spine Surgery: Transvertebral Anterior Cervical Foraminotomy and Posterior Foraminotomy. J Am Acad Orthop Surg Glob Res Rev 2018;2:e008.

31. Deib G, Johnson A, Unberath M, et al. Image guided percutaneous spine procedures using an optical seethrough head mounted display: proof of concept and rationale. J Neurointerv Surg 2018;10:1187-91.

32. Yoon JW, Chen RE, Han PK, et al. Technical feasibility and safety of an intraoperative head-up display device during spine instrumentation. Int J Med Robot 2017;13.

33. Agten CA, Dennler C, Rosskopf AB, et al. Augmented Reality-Guided Lumbar Facet Joint Injections. Invest Radiol 2018;53:495-8.

34. Carl B, Bopp M, Sass B, et al. Implementation of augmented reality support in spine surgery. Eur Spine J 2019;28:1697-711.

35. Carl B, Bopp M, Sass B, et al. Augmented reality in intradural spinal tumor surgery. Acta Neurochir (Wien) 2019;161:2181-93.

36. Carl B, Bopp M, Sass B, et al. Reliable navigation registration in cranial and spine surgery based on intraoperative computed tomography. Neurosurg Focus 2019;47:E11.

37. Müller F, Roner S, Liebmann F, et al. Augmented reality navigation for spinal pedicle screw instrumentation using intraoperative 3D imaging. Spine J 2020;20:621-8.

38. Yoon JW, Chen RE, Kim EJ, et al. Augmented reality for the surgeon: Systematic review. Int J Med Robot 2018;14:e1914.

39. Guha D, Alotaibi NM, Nguyen N, et al. Augmented Reality in Neurosurgery: A Review of Current Concepts and Emerging Applications. Can J Neurol Sci 2017;44:235-45.

40. Chakravarthy V, Sheikh S, Schmidt E, et al. Imaging Technologies in Spine Surgery. Neurosurg Clin N Am 2020;31:93-101.

41. Pfandler M, Lazarovici M, Stefan P, et al. Virtual realitybased simulators for spine surgery: a systematic review. Spine J 2017;17:1352-63.

Cite this article as: Yuk FJ, Maragkos GA, Sato K, Steinberger J. Current innovation in virtual and augmented reality in spine surgery. Ann Transl Med 2021;9(1):94. doi: 10.21037/atm-20-1132 